INPLASY

PROTOCOL

To cite: Pan et al. Prognostic role of pretreatment C-reactive protein to albumin ratio in metastatic colorectal cancer: A systematic review and metaanalysis. Inplasy protocol

202120013. doi:

10.37766/inplasy2021.2.0013

Received: 02 February 2021

Published: 03 February 2021

Corresponding author:

Yan Pan

pyyzrx1985@163.com

Author Affiliation:

Fuyang First People's Hospital, Hangzhou, Zhejiang, China

Support: None.

Review Stage at time of this submission: The review has not yet started.

Conflicts of interest:

None declared.

\section{Prognostic role of pretreatment C- reactive protein to albumin ratio in metastatic colorectal cancer: A systematic review and meta-analysis}

Pan, Y1; Wang, L2; Lou, Y3.

Review question / Objective: This meta analysis is performed to assess the prognostic role of the pretreatment C-reactive protein-to-albumin ratio in metastatic colorectal cancer. P: metactatic colorectal cancer; O: Overall survival, recurrencefree survival, disease-free survival; S: Observational study. Condition being studied: We divided metastatic colorectal cancer patients into high pretreatment $\mathrm{C}$-reactive protein-toalbumin ratio(CAR) group and low CAR group to find the prognosis role of CAR.

Information sources: A comprehensive literature research was conducted on Pubmed, Cochrane library and Embase databases. The search terms used were as follows: ("Creactive protein to albumin ratio" OR "C-reactive protein/ albumin ratio" OR "C-reactive protein albumin ratio" OR "Creactive protein-to-albumin ratio") AND ("carcinoma" OR "neoplasm" OR "tumor" OR "cancer") AND ("colorectal" OR "rectal" OR "colon") AND ("survival" OR "death" OR "mortality") AND ("metastases" OR "metastatic"). Reference lists of included articles and pertinent reviews were also manually searched to supply data. The language was not restricted.

INPLASY registration number: This protocol was registered with the International Platform of Registered Systematic Review and Meta-Analysis Protocols (INPLASY) on 03 February 2021 and was last updated on 03 February 2021 (registration number INPLASY202120013).

\section{INTRODUCTION}

Review question / Objective: This meta analysis is performed to assess the prognostic role of the pretreatment $\mathrm{C}$ reactive protein-to-albumin ratio in metastatic colorectal cancer. P: metactatic colorectal cancer; O: Overall survival, recurrence-free survival, disease-free survival; S: Observational study.

Condition being studied: We divided metastatic colorectal cancer patients into high pretreatment C-reactive protein-to- 
albumin ratio(CAR) group and low CAR group to find the prognosis role of CAR.

\section{METHODS}

Participant or population: Patients with metastatic colorectal cancer (confirmed by histopathology).

Intervention: High CAR group.

Comparator: Low CAR group.

Study designs to be included: Both retrospective study and prospective study can be included.

Eligibility criteria: Inclusion criteria for selecting the studies for this meta-analysis were as follows: (i) Studied patients with metastatic colorectal cancer were pathologically confirmed; (ii) CAR was measured by serum based methods; (iii) Correlation of CAR with overall survival (OS); (iv) The study type of article should be cohort study. Exclusion criteria were as follows: (i) Abstracts, letters, case reports, reviews or nonclinical studies; (ii) Studies with insufficient data for estimating hazard ratio (HR) and $95 \%$ confidence interval (Cl); (iii) Studies had duplicate data or repeat analysis; (iv) Full text cannot be obtained.

Information sources: A comprehensive literature research was conducted on Pubmed, Cochrane library and Embase databases. The search terms used were as follows: ("C-reactive protein to albumin ratio" OR "C-reactive protein/albumin ratio" OR "C-reactive protein albumin ratio" OR "C-reactive protein-to-albumin ratio") AND ("carcinoma" OR "neoplasm" OR "tumor" OR "cancer") AND ("colorectal" OR "rectal" OR "colon") AND ("survival" OR "death" OR "mortality") AND ("metastases" OR "metastatic"). Reference lists of included articles and pertinent reviews were also manually searched to supply data. The language was not restricted.

Main outcome(s): The hazard ratio (HRs) of CAR and the corresponding $95 \% \mathrm{Cls}$ overall survival (OS) or recurrence-free survival (RFS) or disease-free survival (DFS) in metastatic colorectal cancer.

Quality assessment / Risk of bias analysis: Begg's funnel plot and the Egger's linear regression test were performed to evaluate publication bias. The NOS consists of three parts: selection (0-4 points), comparability (0-2 points), and outcome assessment (0-3 points). NOS scores of 6 were assigned as high-quality studies.

Strategy of data synthesis: We directly obtained HR and $95 \% \mathrm{Cl}$ from each literature or estimated these data according to the data obtained from survival curves with Engauge Digitizer and an application based on the method of Jayne F Tierney. HR>1 indicated a worse prognosis metastatic colorectal cancer patients with high expression of CAR. Cochran's $Q$ test and Higgins I-squared statistic were undertaken to assess the heterogeneity of the included trials. Random effects models were used to calculate the pooled HRs and $95 \% \mathrm{Cls}$ because of the data included had heterogeneity. Subgroup analysis was conducted to explore and explain the heterogeneity among the results of different studies. Publication bias was assessed by Begg's and Egger's funnel plot. All $p$-values were two-sided. A p $<0.05$ was considered statistical significant. All the statistical analyses were performed using Stata statistical software version 12.0.

Subgroup analysis: Subgroup analysis was conducted to explore and explain the heterogeneity among the results of different studies. If there is heterogeneity, we will divide the study into several different subgroups based on the sample size, nation, cut-off value of CAR and so on.

Sensitivity analysis: We will use STATA for sensitivity analysis.

Country(ies) involved: China. 
Keywords: metastatic colorectal cancer; Creactive protein to albumin ratio; prognosis; meta-analysis

Contributions of each author:

Author 1 - Yan Pan.

Email: pyyzrx1985@163.com

Author 2 - Lin Wang.

Email: dongting2010zygs@163.com

Author 3 - Yinmei Lou.

Email: lymyzrx@163.com 